\title{
Sol: THE NITROGEN CYCLE IN NATURE
}

$\mathrm{O}$ $\mathrm{N}$ September 2, a meeting in Nefroastle, a discussion was held by Section B (Chpraisty) on "The Nitrogen Cycle in Nature". Prot G. R. Clemo, in introducing the discussion, hate a/general survey of the great importance of the rittogen cycle in Nature, which en îres fhàt, filit obther natural resources generally, our jyast reser res of atmospheric nitrogen are never likely to becomexhausted. Among the many groups of naturally occurring nitrogenous substances, he made special mention of chloromycetin, the first organic nitro compound to be discovered in Nature and containing the fundamental $\mathrm{C}_{6} \mathrm{C}_{3}$ unit characteristic of many plant products.

Robinson's earlier ideas on the biogenesis of alkaloids have recently been followed up, leading to new ideas on the structure of emetine and to the synthesis under physiological conditions of sparteine by Anet, Hughes and Ritchie of Sydney. Prof. Clemo also made reference to possible future developments, including work on bee venom, which is stated to contain a specific protein or proteins and to have a bearing on rheumatic diseases.

Prof. A. I. Virtanen gave an account of the work being carried out at Helsinki by himself and his collaborators on the utilization of the nitrate ion by plants and its relation to the assimilation of the ammonium ion and molecular nitrogen. For an understanding of the mechanism of the assimilation of nitrogen, one of the important questions is whether nitrate is reduced to ammonia or whether ammonia is the central nitrogenous compound which is produced from all nitrogen sources (including molecular nitrogen) before amino-acid synthesis. The one observation against this thesis is the formation of oxime nitrogen; its mode of formation is therefore a problem of great interest. A simple chemical explanation of $\alpha$-amino-acid formation might, of course, be the action of hydroxylamine on $\alpha$-ketoacids, if the former were, in fact, present.

With Torula, it has been shown that oxime nitrogen arises from nitrate in strongly aerated solutions, very rapidly reaching the maximum within 10-15 min. and then diminishing. In a corresponding experiment, ammonium nitrogen does not yield oxime nitrogen. Similarly, Virtanen and Jarvinen have shown that, in comparable experiments, Azotobacter produces oxime nitrogen more rapidly from molecular nitrogen and from nitrate nitrogen (about 1-2 hr. in each case to obtain a detectable amount of oxime) than from ammonium nitrogen ( $3 \mathrm{hr}$.), although in the latter case the induction period is absent. These results suggest that, both in the reduction of nitrate and in the fixation of molecular nitrogen, oxime nitrogen is formed before ammonia, rather than after it, although the reduction to ammonia is probably the main process :

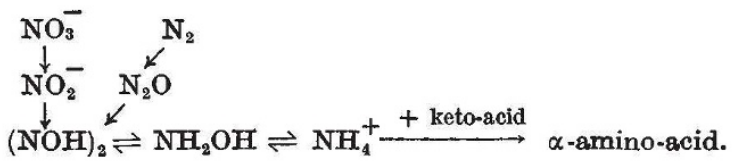

In anaerobic nitrogen fixation by Clostridium, Virtanen and Hakala failed to detect any traces of oxime nitrogen. The anaerobic nitrogen fixation is most likely to proceed via reduction (cf. Wieland). In the aerobic nitrogen fixation a primary oxidation of nitrogen is possible. From the $(\mathrm{NOH})_{2}$ stage onwards, reduction of nitrate and fixation of molecular nitrogen would then follow the same path.

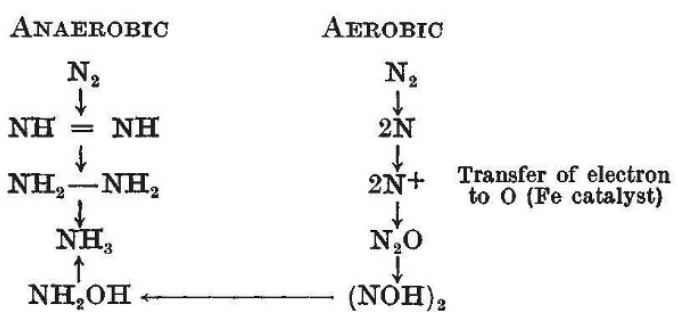

The postulation of these two different routes is not attractive but, at present, seems unavoidable. All the results so far obtained imply that all nitrogen sources produce chiefly ammonium nitrogen prior to amino-acid synthesis; but that hydroxylamine, which is formed in nitrate reduction and in aerobic nitrogen fixation, yields also small amounts of oxime nitrogen. The cells ought, therefore, also to have an enzyme system to catalyse the latter route. Oxidation of ammonium nitrogen leads likewise to oxime nitrogen.

Nitrate reduction has generally been noted to be sensitive to cyanide, and hence a heavy metal would be an essential factor in the enzyme system. Warburg first suggested that the metal might be iron; but later Burstrom thought manganese more likely. Molybdenum, too, seems to be indispensable in the assimilation of nitrate as well as, according to Mulder, that of molecular nitrogen. Wirth and Nord have found that nitrate reduction with Fusarium is not sensitive to cyanide, and hence nitrate reduction can be brought about by different enzyme systems.

Finally, Prof. Virtanen described a recent extension which follows on from work he had done before the War on the growth of green plants (for example, peas) from seeds the cotyledons of which had been removed. In these experiments, nitrate was used as the nitrogen source, and it was shown that addition of ascorbic acid could restore normal growth (the ascorbic acid of pea seeds is normally found mainly in the cotyledons). It is now found that for growth on media containing ammonia no ascorbic acid is needed-in fact, if added, it actually retards the growth. Similar results have been obtained with endosperm-free wheat germs. That the effect (for growth on nitrate) is not specific for ascorbic acid but is, in fact, brought about by other reducing substances (for example, sodium sulphide, cysteine, glutathione, reductone) has been shown by Virtanen and Saubert-v. Hausen. Although Karrer has shown that ascorbic acid will reduce nitrite in boiling solution, this requires an acid solution and is unlikely to occur in the plant cell at $p \mathbf{H ~} 6$, and, moreover, no accumulation of nitrite has been observed in the cotyledonless plants. Thus, the effect of these substances is not based on their ability to reduce nitrate, but is associated with the function of the nitrate. reducing enzyme system. 
Dr. G. R. Tristram then dealt with the work of Chibnall, Vickery and other workers on the aminoacid/protein aspect of the nitrogen cycle. Within the main nitrogen cycle there is another cycle involving a turnover between protein, amino-acid and ammonia, and it is important to realize that this cycle does not operate merely as more protein is required but that there is a continuous elaboration and breakdown of protein. The problem discussed was the mechanism by which nitrogen and various carbon skeletons are united with the ultimate formation of protein.

Whether or not the amino-acids are the actual intermediates in the synthesis of protein, it is now well established that the dicarboxylic amino-acids, glutamic and aspartic acids, and their respective amides, are the key compounds in its elaboration, not only as a ready source of amino-groups, but also, in the case of glutamic acid, as a source of a carbon skeleton. Dr. Tristram stressed the interconversion of carbon skeletons as a mechanism for the synthesis of amino-acids, as well as merely the donation of amino-groups on to a carbon skeleton from another source. In addition, both these amino-acids and alanine provide a connexion with carbohydrate meta. bolism. That glutamic acid is the first product synthesized in the assimilation of ammonia or nitrate was shown by Burris and Wilson by experiments in which $\mathrm{N}^{1} \mathrm{H}_{3}$ was fed to certain micro-organisms. Amino-acids may arise in a number of different ways, for example, by the amination of carbonyl compounds (for example, keto-acids) with ammonia or hydroxylamine, by the addition of ammonia to unsaturated acids, such as fumaric acid, or by transamination, in which case the amino-group is transferred from an amino-dicarboxylic acid to a keto-acid. The latter was originally thought to be a general reaction involving glutamic acid and the keto analogue of any amino-acid. It is now known that it is relatively restricted as a fast reaction involving glutamic acid, oxalacetic acid and pyruvic acid. Glutamic acid, plus ammonia, is converted into its amide with the aid of adenosine triphosphate. There is no evidence to show that glutamine and asparagine are synthesized by glutaminase and asparaginase working in reverse. The mechanism of asparagine synthesis is not known.

One role of the amidases is to store, in non-toxic form, the ammonia which is liberated on protein breakdown, and thus to conserve nitrogen. One fact which emerges from the vast amount of work on amide synthesis in plant tissue is that glutamine is always present, although sometimes in very small amount, whereas asparagine may be completely absent. This may mean that glutamine is more important as an intermediary than is asparagine, which would function merely as a store of aminogroups.

The fact that the keto analogues of certain of the so-called essential amino-acids are effective for growth in animals suggests that their carbon skeletons might be the parts of the molecule which cannot be synthesized. Using an analogy from animal work, Chibnall put forward the view that these carbon skeletons might well arise from the organic acids some of which take part in the Krebs cycle, suggesting a close link with carbohydrate metabolism. Nevertheless, the origin of the carbon skeletons of valine, leucine, isoleucine, tyrosine and phenylalanine is still to be explained.

As to the mode of protein synthesis, many theories have been advanced. Although protein synthesis may well involve the preliminary formation of amino-acids followed by their condensation into peptide linkages, there is no conclusive evidence that protein synthesis is, in fact, the reverse of proteolysis. Dr. Tristram then outlined the theories of du Vigneaud, Linderstrøm-Lang and Bergmann and Fruton, and pointed out that they lacked experimental evidence to support them. He suggested that it is possible that there are several different mechanisms for peptide formation of the various amino-acids (for example, lysine in animals must be fed as such, but methionine can be fed as the keto analogue).

Consequently it is possible that peptide synthesis involving methionine keto-acid may be different from a synthesis involving Iysine. Dr. Tristram then pointed out the view of himself and Mr. Hird that it may be possible that the keto analogue is aminated and coupled in a single stage to form a peptide linkage with the aid of a labile amino-donating group and phosphate energy. This could account for the amination of amino-acids which do not appear to participate in transamination.

In the succeeding paper, Prof. J. H. Quastel dealt with the effects of organic nitrogen compounds (especially amino-acids) on soil nitrification, as studied by the use of his own simple and elegant soil perfusion technique, a brief account of which was given. The bearing of hydrogen ion concentration on the process of nitrification was discussed, and it was shown that organic molecules which yield ammonia are more readily nitrified by an acid soil than are the inorganic ammonium salts. Similarly, non-nitrogenous organic molecules which yield bicarbonate as a product of their metabolism assist nitrification in acid soils. On neutral or alkaline soils the inorganic ammonium salts are nitrified a little more readily than are the amino-acids.

All the amino-acids studied nitrify readily, with the striking exception of methionine. If methionine is mixed with ammonium chloride, nitrification is retarded for sixteen days, and then suddenly both are nitrified very rapidly. This is the only known naturally occurring nitrogen compound which inhibits nitrification. It also delays the conversion of nitrite to nitrate for ten days; but the methionine is itself in this case unattacked, indicating that the organisms have developed a tolerance. The presence of the amino-acid in proteins and protein decomposition products may account, partly at any rate, for the inhibitory action of these substances on nitrification. The amino-acids studied do not yield all their nitrogen in the form of nitrate; the yield is inversely proportional to the carbon/nitrogen ratio of the amino-acid. But they do not, with the exception of methionine, inhibit the nitrification of ammonium ions. The mechanism of action of methionine may give a clue to the manner in which ammonia undergoes metabolism in nitrifying cells.

Thiourea also inhibits nitrification of ammonia, even at as low a concentration as $0.0003 M$, for at least sixteen days. Ethyl urethane $(0 \cdot 01 M)$ inhibits nitrification for about three weeks and then is itself nitrified through the development of a special urethanemetabolizing organism. These substances presumably interfere in some way with some aspect of ammonia metabolism, and it is interesting to note that they are both carcinogens. The metabolism of enriched soil has also been investigated in the Warburg apparatus; the rate of oxygen uptake using ammonium chloride is linear; and thiourea lowers 


\section{No. 4176 November 12, $1949 \quad$ N A T U R E}

the rate, but a much higher concentration is here needed.

Lastly, Prof. Quastel discussed his work on the metabolism of pyruvic oxime. This is nitrified very rapidly in soil into nitrite and nitrate. The effect can readily be demonstrated by the addition to enriched soil first of hydroxylamine, which is toxic ; but on the subsequent addition of pyruvic acid, rapid nitrification ensues. Other oximes (except that of oxalacetic acid) do not show comparable effects. Also, methionine is without effect, showing that this direct conversion of pyruvic oxime into nitrite is not accomplished by Nitrosomonas or Nitrobacter but by a class of heterotrophic soil organisms which have been isolated. This result shows that nitrification in soil may not be accomplished wholly by the well. known autotrophic organisms but that heterotrophic organisms may play a part in the direct nitrification of an organic nitrogen molecule, although there is, as yet, no proof that pyruvic oxime is a normal meta. bolic product under soil conditions.

The concluding paper was read by Dr. E. J. Hewitt and was concerned with the importance of molybdenum in the nitrogen cycle-a subject already touched upon by Prof. Virtanen-which is being studied at Long Ashton. Molybdenum is of widespread significance in the plant kingdom. It is needed in certain circumstances by free and symbiotic nitrogen-fixing and denitrifying organisms, by fungi and by representatives of at least ten families of higher plants. The minute amounts of molybdenum needed have rendered progress slow, until recently, when special techniques have been devised. Deficiency and excess symptoms in higher plants suggest that molybdenum influences nitrogen metabolism, meristematic development and water relations. Brassica types especially and tomato and clovers seem to be particularly susceptible. Molybdenum is essential for Azotobacter and Rhizobium when fixing nitrogen. It is also needed by higher plants and by Aspergillus niger, denitrifying bacteria and Azotobacter when supplied with nitrate nitrogen, but apparently it is not needed at all (or at greatly reduced levels) when nitrogen is given as ammonia or urea. When molybdenum is lacking, nitrate accumulates or persists and is not reduced. Vanadium can replace molybdenum at a low level of efficiency in Azotobacter, but not in Rhizobium or in the metabolism of fungi and higher plants. The precise step in reduction of nitrogen compounds catalysed by molybdenum is still obscure.

Manganese is also essential for nitrogen assimilation, since nitrate accumulates when manganese is deficient. Manganese, however, has an opposite effect on amino-acid concentration; but the effects due to its deficiency are also partly dependent on the source of nitrogen.

Soil fertility may be influenced by the availability of the molybdenum. This element is less available in acid soils, and such soils are frequently deficient in available nitrogen. Failures in the establishment of subterranean clovers and other legumes in poor acid pastures of South Australia have been traced to unavailability of molybdenum for the root nodule organisms. Such pastures appear nitrogen-deficient, and beneficial results are obtained by application either of large amounts of sodium nitrate or as little as a quarter of an ounce per acre of sodium molybdate. As clovers given nitrate nitrogen in culture experiments still require molybdenum, it is evident that when given sodium nitrate in soil there is sufficient molybdenum for the requirements of the plant, although not enough for those of the root nodule bacteria. In spite of this differential effect on clovers, Brassica types may still suffer from molybdenum deficiency in the field; and the condition long known as 'whiptail' in broccoli and cauliflower may be related to molybdenum deficiency and has been reproduced experimentally in sand cultures by omitting molybdenum. Liming greatly increases the availability of molybdenum; its effect may be on $p \mathrm{H}$ directly or on reducing the availability of manganese (the two elements apparently being antagonistic).

Molybdenum is thus important in three aspects of the nitrogen cycle, namely, fixation, assimilation from nitrate and denitrification; but in all a reduction process seems to be involved.

Following the reading of the papers, a discussion took place during the afternoon, which emphasized the very incomplete present-day state of knowledge of the chemical reactions underlying the nitrogen cycle.

G. R. CLFmo

G. A. SWAN

\section{CONSERVATION $\% \%$ AND UTILIZATION OF WORLD RESOURCES}

\section{UNITED NATIONS CONFERENCE}

$\mathrm{D}^{\mathrm{u}}$ URING the period August 17-September 6, the United Nations Scientific Conference on the Conservation and Ufilization of Resources, convened by the Sropian and Economic Council, was held at Lake Succeds, New York. Its deliberations covered a very fide field, in that they dealt with the conser toxion am d utilization of the principal resources affeting (ijman life throughout the world, namely, minerals, fuels and energy, water, forests and land. The Conference was attended by 535 delegates from forty-nine nations, which included various Eastern European countries but not the U.S.S.R.

The Conference was the first one on world conservation ever to be held. It was a first step by the United Nations towards mobilizing the science of the whole world to promote higher standards of living, and towards implementing the programme of tech. nical assistance to under-developed countries which the United Nations Organisation is undertaking. By such means, it is hoped to bring into sustained use resources as yet untapped and to build up more productive and diversified economics. Stress was laid on the part to be played by major concerted undertakings wherever possible.

The Conference was scientific rather than policy. making. It had no power to bind governments, and did not formulate recommendations to them, nor did it attempt to reach international agreements on policies; its task was limited to the exchange of experiences on the techniques of the conservation and utilization of resources. In the course of discussions, however, both formal and informal, questions of policy inevitably crept in, since it is practically impossible to consider the broader problems of conservation separately from those of control. It was generally conceded that in the more compre- 